\title{
Animal salmonelloses: a brief review of "host adaptation and host specificity" of Salmonella spp.
}

\author{
Grammato Evangelopoulou', Spyridon Kritas ${ }^{2}$, Alexander Govaris ${ }^{3}$ and Angeliki R. Burriel ${ }^{1}$
}

\begin{abstract}
1. Laboratory of Microbiology and Parasitology, Faculty of Veterinary Medicine, School of Health Science, University of Thessaly, Trikalon 224, Karditsa 43100, Greece, Tel.: +30-2441066088, Fax: +30-2441066088, e-mail: matinavet@hotmail.com; 2. Department of Microbiology and Infectious Diseases, School of Veterinary Medicine, Aristotle University, 54124 Thessaloniki, MKD, Greece; 3. Laboratory of Hygiene of Foods of Animal Origin,

Faculty of Veterinary Medicine, School of Health Science, University of Thessaly, Trikalon 224, Karditsa 43100, Greece, e-mail: agovaris@vet.auth.gr

Corresponding author: Grammato Evangelopoulou, e-mail:matinavet@hotmail.com

Received: 20-05-2013, Revised: 05-06-2013, Accepted: 05-06-2013, Published online: 31-07-2013
\end{abstract}

doi: $10.14202 /$ vetworld.2013.703-708

How to cite this article: Evangelopoulou G, Kritas S, Govaris A and Burriel AR (2013) Animal salmonelloses: A brief review of "Host Adaptation and Host Specificity" of Salmonella spp., Veterinary World 6(10): 703-708.

\begin{abstract}
Salmonella enterica, the most pathogenic species of the genus Salmonella, includes more than 2,500 serovars, many of which are of great veterinary and medical significance. The emergence of food-borne pathogens, such as Salmonella spp., has increased knowledge about the mechanisms helping microorganisms to persist and spread within new host populations. It has also increased information about the properties they acquire for adapting in the biological environment of a new host. The differences observed between serovars in their host preference and clinical manifestations are referred to as "serovar-host specificity" or "serovar-host adaptation". The genus Salmonella, highly adaptive to vertebrate hosts, has many pathogenic serovars showing host specificity. Serovar Salmonella Typhi, causing disease to man and higher primates, is a good example of host specificity. Thus, understanding the mechanisms that Salmonella serovars use to overcome animal species' barriers or adapt to new hosts is also important for understanding the origins of any other infectious diseases or the emergence of new pathogens. In addition, molecular methods used to study the virulence determinants of Salmonella serovars, could also be used to model ways of studying the virulence determinants used by bacteria in general, when causing disease to a specific animal species.
\end{abstract}

Keywords: adaptation, evolution, host, Salmonella

\section{I ntroduction}

Salmonella spp., belonging to the family of Enterobacteriaceae, are Gram negative, facultative anaerobic, straight rods, with peritrichous flagella. The genus includes the species $S$. enterica (the type species) and $S$. bongori, which are divided into more than 2,500 serovars. Worth noting is that, the previously known subspecies of $S$. enterica referred to as subspecies I, II, IIIa, IIIb, IV and VI are currently named as $S$. enterica subsp. enterica (subspecies I), $S$. enterica subsp. salamae (subspecies II), S. enterica subsp. arizonae (subspecies IIIa), S. enterica subsp. diarizonae (subspecies IIIb), S. enterica subsp. hountanae (subspecies IV) and S. enterica subsp. indica (subspecies VI) [1]. The genus Salmonella, recovered from nearly all vertebrates and many insects, is considered a "universal pathogen" [2]. The pathogenic specificity of Salmonella serovars depends on each serovar's ability to adapt to the environment of its host. This adaptation is regulated by various microbial characteristics, which also assist the expression of specific clinical manifestations in a specific host species. In addition to the virulence determinants of a serovar, important are also the

Copyright: The authors. This article is an open access article licensed under the terms of the Creative Commons Attribution License (http://creativecommons.org/licenses/by/2.0) which permits unrestricted use, distribution and reproduction in any medium, provided the work is properly cited. infectious dose, the animal species infected, their age and individual immune response [3]. They and, perhaps, many other, yet unknown, virulence determinants are molecularly studied, as new pathogenic Salmonella serovars emerge. The accumulated evidence show that a mechanism making a serovar virulent for one animal species could make this serovar less or completely avirulent for another animal species. This acquired phenomenon is called "serovar host specificity" or "serovar host adaptation" [4]. Two Salmonella examples indicative of host specificity in animals are Salmonella enterica ser. Dublin, usually associated with disease in cattle, and Salmonella enterica ser. Choleraesuis consistently responsible for only pig salmonellosis [5]. However, regardless of the accumulated information on host specificity and adaptation, a clear understanding is yet to be achieved on a serovar's pathogenicity, with regard to an animal host.

Nevertheless, newer and older molecular methods used to epidemiologically study serovars have shown close DNA relatedness between serovars and a common ancestor living 25 to 40 million years ago $[4,6,7]$. These findings demonstrate that Salmonella spp. has, since its divergence from the $E$. coli lineage, most likely evolved in three phases [6].

In the first phase the microorganism acquired, through horizontal gene transfer, the SPI-1 (Salmonella 
Pathogenic Island-1) pathogenicity determinant. The SPI-1 encodes genes helping the microorganism to invade host cells [8] and it is present in all Salmonella serovars, but absent in E.coli and other relative bacteria.

During the second phase, two distinct species of Salmonella emerged by horizontal gene transfer. They are Salmonella enterica acquiring also the SPI-2, which is helping it to survive within host cells, such as the macrophages [9] and Salmonella bongori. Both species are possessing pathogenic determinants helping them to colonize deeper tissue. In addition to the two major virulence determinants of $S$. enterica (SPIs 1 and 2), there are also 14 different pathogenicity islands present in varying numbers within different serovars [10].

The third phase of the microorganism's evolution is related to the division of Salmonella spp. into subspecies and the adaptation of subspecies I (subspecies enterica) to warm-blooded vertebrates (birds and mammals), thus adaptation within an increased range of animal hosts. The remaining subspecies and Salmonella bongori are mainly adapted to cold-blooded vertebrates. Therefore, one couldn't but question the nature of the mechanisms helping Salmonella serovars diverge from their common ancestor and emerge as serovars differing in their host range.

For understanding the many biological characteristics leading to serovar development, essential is the understanding of the mechanisms helping a particular serovar to adhere and proliferate in the tissue of a given host. Thus, research has focused on the microorganism's acquired ability for "host specificity and adaptation".

\section{Host specificity - host adaptation of the genus Salmonella}

The majority $(99.5 \%)$ of the isolated serovars from animals and man belong to $S$. enterica subsp. enterica. The other subspecies are associated mainly with cold-blooded animals. All animal species are susceptible to various Salmonella serovars under certain circumstances, but not all are as severely affected clinically. Clinical severity is associated with specific serovars affecting specific animal species [11]. Most of the serovars belonging to subspecies enterica cause acute, localized gastroenteritis of short duration rather than systematic disease. Only a small number of serovars are causes of severe systemic disease in man and animals, manifested as septicaemia, fever and/or abortion. The latter serovars, although due to their limited number are extensively studied molecularly, they haven't yet fully revealed the factors of their adaptation [4].

Scientifically, all pathogens could, perhaps, be considered as host-adapted pathogens, regardless of observed epidemiological differences between similar microorganisms showing some as having a single host susceptible to them and others having a greater number. Thus, attempting to define the importance of "host specificity-host adaptation" in Salmonella infections, the properties of pathogenicity characterizing a specific serovar, epidemiologically observed in a sensitive host, should be defined. Such attempts of defining the pathogenicity determinants of Salmonella serovars causing infections in man and animals have resulted in grouping serovars in three major groups.

One group includes serovars, such as Dublin and Choleraesuis, proven to cause systemic disease respectively in cattle and pigs only, while other animal species or man could be infected accidentally $[12,4]$. However, in the latter hosts, infection is frequently subclinical, making them carrier animals, thus "symptomless excreters" and reservoirs of the infecting serovars. These animals cause environmental contamination, becoming a risk to susceptible animal species [4].

The second group includes serovars, such as Typhi, Gallinarum, Abortusovis, Typhisuis and Abortusequi, which are highly host adapted, thus, they are almost exclusively associated with systemic disease when they infect man, fowl, ovine, pigs and equine, respectively. These serovars, referred to as host-restricted (HR) serovars, are strictly associated with one very specific host species $[3,13]$.

The third group of serovars consists of non-host adapted serovars called unrestricted serovars posing a risk to many animal species and man. The most clinically important serovars in this group are Salmonella Typhimurium and Salmonella Enteritidis. They are of a greater epidemiological importance, compared to the previous ones, although they cause a relatively mild enteric disease to their hosts [13, 14]. These serovars are also associated with more severe disease in young hosts, compared to adults. Perhaps, they lack mechanisms helping them to invade the mature immune system of older hosts [6].

In summarizing the above, host adaptation in many ways, is the ability of a pathogen to cause disease in a particular animal population, regardless of the degree of pathogenicity it exhibits for a different animal host. For example, serovar Choleraesuis is considered a porcine adapted serovar, not because it causes the severest disease in swine compared to man, but because it persists in pig populations infected by direct transmission, making them a natural host for this serovar, thus its reservoir [5]. What are, therefore, the known factors determining "host specificity and adaptation"?

\section{Factors within the genus Salmonella determining host specificity and adaptation}

Although the exact mechanisms leading to "host specificity" have not been fully understood, the existing evidence shows that they act independently of each other at the various phases of an infection. During the process of adaptation, the expression of a serovar's 
pathogenicity is influenced by the environmental and genetic factors influencing each host [15]. During the course of this adaptation, each host adapted/host restricted serovar (HA/HR) must overcome the encountered specific and non-specific immune mechanisms. Thus, pathogenicity of HA serovars results from the development of ways helping their survival in a host. Examples to this are serovars of Salmonella enterica subsp. enterica, which have developed the ability to evade the immune mechanisms of warm-blooded animals. They have, during their evolution, acquired the ability to modify to their favor, the physiological functions of their host, such as intracellular engulfment, apoptosis, transfer of antigens by $\mathrm{M}$ cells, migration of macrophages and lymphocytes in the reticuloendothilial system and others [6]. A well known serovar belonging to this group of serovars, is Salmonella Typhi, pathogenic to man, but not, for e.g., to mice. Its acquired ability to survive and divide in only human macrophages, makes it pathogenic to man, but not to mice $[16,17]$. Serovars such as the HR Salmonella Typhi, Salmonella Gallinarum and Salmonella Abortusovis, showing high tropism for the lymphatic organs of their hosts, are, perhaps, regulating their natural hosts' biological environment, in their favor [15]. They, by attaching to cells of the bone marrow, bursa of Fabricious and Peyer's patches are affecting the development of Bcells, thus immune response. The result of such interactions, particularly in adult animals, help the establishment of chronic or subclinical infection and, thus prolonged subclinical excretion, but they do not help the development of severe gastroenteritis $[18,19]$. Serovars not fully adapted to evade mature immune system, lack specificity, causing deadly systemic disease in adult animals by invading their defense mechanisms compared to HR serovars [6]. The latter are mildly enteropathogenic compared to the unrestricted serovars, thus, they do not cause intestinal inflammation [20]. A common characteristic of HR serovars is their ability to infect the reproductive system, affecting egg production in poultry and causing abortions in mammals. They greatly proliferate in fetal tissues, which when aborted, disseminate a large number of microorganisms in the environment [3].

It has also been shown that the ability of a serovar to metabolize a wide range of amino acids adds to its virulence $[21,22]$. Although, serovars metabolizing for their multiplication the same metabolites (e.g. cysteine, thiamin, nicotinic acid) are thought as closely related, each HR or HA serovar has most likely evolved independently. On the other hand, the heterogenicity of serovars in relation to different metabolic profiles, facilitates either the completion of the pathway to infection [23] or, when lacking specificity, it is favoring host adaptation [15].

The process of Salmonella host adaptation is believed that either it involves acquisition of novel genetic elements encoding specific virulence factors or loss of genes, thus a liability observed frequently in pathogenic strains. Best examples of host specificity dependent on gene deletions are, perhaps, serovars Salmonella Typhimurium, Enteritidis, Choleraesuis, Gallinarum, Pullorum, Abortusovis, Paratyphi $\mathrm{C}$ and Dublin [14]. Studies using genome sequencing of hostadapted/host-restricted serovars, such as Typhi, Gallinarum, Choleraesuis and the newly emerging in sub-Saharan Africa invasive strains of Salmonella Typhimurium, revealed that these serovars have undergone extensive gene deletion and truncation [2428]. In systemically non-invasive Salmonella, the majority of lost genes have functional orthologs, which play a key role in intestinal colonization. Thus, these lost genes have resulted in loss of an intestinal multiplication cycle for narrowly host adapted Salmonellae followed by a concurrent acquisition of mechanisms helping the microorganism to survive in a systemic niche [27]. Point mutations, the target of positive selection, together with horizontal gene transfer and genome degradation could be responsible for a differential pathoadaptive evolution of some Salmonella serovars [29]. It appears from the analysis of the mannosesensitive fimbrial adhesin FimH that even single amino acid replacement, resulting in specific structural mutations in FimH variants of host-adapted (systemically invasive) serovars, play an important role in the differential adaptive evolution of Salmonella spp. Thus, activation or inactivation of mannose-specific adhesive properties in different systemically invasive serovars of Salmonella reflects the dynamic trajectories of adaptation of a serovar to the biological environment of a specific host. Furthermore, phylogenetic analysis has indicated that these mutations are, most likely, of a convergent nature (common pathogenic traits incorporated into different genetic backgrounds) and occur under strong positive selection, illustrative of the role of point amino acid changes for a hostadapted Salmonella.

Certainly, in depth study is needed for the molecular composition of flagella, chemotaxis genes [30], fimbriae, bacteriophages, the presence of virulence plasmids and subtypes of each specific serovar, to understand mechanisms of pathogenicity and host specificity $[14,15]$. Studies correlating some phage types of Salmonella Typhimurium with their hosts have shown marked host specificity [31]. However, the majority of phage types studied had a broad host range, perhaps, suggesting a phage transfer of virulent genes between hosts, leading eventually to host specificity. The unrestricted serovar Typhimurium may comprise a spectrum of variants differing in regard to virulence and reflecting a summation of the spatial and/or temporal selective pressures within a particular host [32]. In this study, all Typhimurium strains deriving from animal clinical cases were virulent also in mice, whereas many Typhimurium strains deriving from man clinically ill lacked this ability. Of interest was that many of the Typhimurium stains deriving from human gastroenteritis lacked the Salmonella virulence plasmid, present in all animal strains and strains 
isolated from human bacteremia. Furthermore, some strains of serovar Typhimurium isolated from man and harboring the Salmonella virulence plasmid were avirulent in mice, and the opposite was observed with those deriving from animals and phenotypically exhibiting virulent determinants. These findings suggest that Salmonella isolates of the same serovar deriving from human salmonelloses are distinctly different from those isolated from animals. This means, that selective pressure within a particular host, may give rise to bacterial strain variants that exhibit different pathogenicity determinants, thus varying degree of pathogenicity.

Similarly, serovars of Salmonella enterica subsp. enterica, associated with disease in mammals and birds, show different degrees of adaptability. Perhaps, pathogenicity determinants, such as the FimH adhesins play an important role. For example, the type 1 FimH adhesins are expressed by serovars of $S$. enterica isolated from mammalian and avian hosts, while the type 2 FimH is expressed exclusively by the avianadapted serovar Gallinarum. Consequently, these data show that allelic variation of the S. enterica FimH adhesin directs host-cell-specific recognition, thus selective binding to mammalian or avian receptors. This allele-specific binding parallels the host specificity of the respective FimH-expressing pathogen [33].

However, regardless of the progress in the study of HA/HR serovars, the mechanisms and genes involved in the expression of "host specificity" are yet to be fully clarified. Research about the distribution of Salmonella Pathogenic Islands (SPIs), fimbriae operons and virulence plasmids has shown that, various combinations of virulent determinants, formed during the evolution of the microorganism, are needed for a variant to become pathogenic in a particular range of host species. Mutations horizontally transmitted could have helped the development of "host specificity" [6] by helping Salmonella serovars to harbour unique virulence factors [34]. Molecular and phylogenetic analyses of the SPIs' genes, showed that these genes encode for translocon proteins (SipD, SseC and SseD) present on both Salmonella pathogenicity islands (SPI-1 and SPI-2) and also encode an effector protein inhibiting the pathway of the host cells mitogen-activated protein kinase (sptP gene) [35]. In addition, they encode effector proteins (SseF and SifA) important in placing the Salmonella-containing vacuole in a juxtanuclear position. The products of these genes directly interact with the host cell and modulate its functions, thus favoring host specificity. Another study of the SPI genes has shown the close evolutionary relatedness between serovars Gallinarum and Enteritidis [36], although the former is highly adapted (restricted) to poultry and is the only known non-motile serovar, while serovar Enteritidis is an unrestricted serovar. Analysis of the functions of genes associated to SPI- 1 to -5 showed that virulence genes might have evolved under positive selection imposed by a serovar's respective host(s) contributing to the different hostspecificity observed between different serovars. Undoubtedly, as information accumulates, a better understanding would be achieved concerning the mechanisms helping closely related serovars to show different host specificities.

This information has revealed that a close similarity of core regions exist, both within and among the genomes of various serovars [37]. Some examples are evidence of recombination and rearrangement, genomic degradation, pseudogenes and clonal diversity. In particular, genomic comparisons of hostrestricted and host-adapted serovars show that genomic degradation is a common evolutionary mechanism for host adaptation and increased pathogenicity [37, 38]. Others have shown that host restriction and change of ecological niche (site of attachment or type of diseases) are linked to the accumulation of large numbers of pseudogenes and an overall reduction in genome size [28]. Examples are serovars Typhi and Paratyphi A, restricted to man and causing a similar systemic disease. Genome sequence similarity between Typhi and Paratyphi A serovars and their different pathogenicity when compared to the unrestricted serovars of $S$. enterica, has been attributed to a relatively recent recombination of a quarter of their genomes, making the accumulation of pseudogenes a key feature of convergent evolution for these and other host-adapted pathogens [29]. Another example supporting the role of convergent evolution among the typhoid agents is serovar Paratyphi $\mathrm{C}$, which has diverged from the same ancestor as serovar Choleraesuis, by accumulating genomic novelty during its adaptation process to man. The genomic analysis of these two Salmonella serovars has revealed a highly similar genomic construction between the two and their distinct pathogenic features, making them excellent models for studying Salmonella's host adaptation and pathogenic divergence [37].

Hence, Salmonella adaptation to a particular host species is a complex phenomenon, which depends, apparently, on a large number of gene products. The mastery of understanding the interactions between Salmonella serovars and their natural hosts needs comparative analysis of the interactions between the physiology of various animal species and their genetic composition.

\section{Conclusions}

Despite the efforts of understanding the fundamental mechanisms of $S$. enterica's pathogenesis, the specific genes involved and the mechanisms needed for a serovar to express "host adaptation-host specificity" are yet to be defined. However, research on the mechanisms involved when pathogens exert selective pressure on the host's immune system has led to the conclusion that "host adaptation" of a serovar helps it to competitively exclude other serovars in a given animal population. In addition, as the degree of 
adaptation increases, evasion of the immune system increases, helping multiplication, thus the increasing excretion of the pathogen, infecting eventually an increasingly larger number of animal species.

Some believe that Salmonella spp. was a commensal microorganism of the reptile gastrointestinal tract long before man appeared and this is why all serovars are easily adapted within herpetofauna, no matter how selective they have become for other animal species [39]. Thus, to better understand the evolution of Salmonella infections, one should study the mechanisms helping the pathogen to be established in a new host. This is possible if "host adaptation-host specificity" are studied during the extensive surveillance and research of animal salmonelloses caused by serovars having a Public Health importance. Those that have attempted it [40] by the analysis of available genome sequences indicate that the broad host range of foodborne serovars is correlated to the maintenance of a wide number of intact genes found in host-restricted or host-adapted variants.

However, the precise nature of a serovar's potential zoonotic and epidemic characteristics is still unknown. The identification of such characteristics is vital for assessing the risk posed to man by S. enterica serovars found in animals, needing effective strategies for protecting Public Health. Improvements in the methods of molecular sequencing can be expected to yield new insights in this area, particularly if applied to epidemic variants and hither to serovars, that although frequently isolated from animals, they rarely appear in man, thus considered of lesser importance [41]. A good understanding of Salmonella's host specificity could also be a useful tool for the designing of autogenus vaccines effective against the prevalent serovars in a farm, thus improving the efficacy of vaccination. Research should, therefore, be focused on the genetic origins of host adaptation and host specificity, enabling researchers to "predict" a serovar's survival in a new animal population, eventually helping them to design the best strategies against serovars causing epidemics in livestock and man.

\section{References}

1. Lin-Hui, S. and Cheng-Hsun, C. (2007) Salmonella: Clinical Importance and Evolution of Nomenclature. Chang Gung Med.J. 30(3): 210-219.

2. Fedorka, P. J. - Cray, T., Gray, J.T, Wray, C. (2000) Salmonella Infections in Pigs In: Wray, C., Wray, A., editor. Salmonella in domestic animals. CABI Publishing, New York. 191-208.

3. Wallis, T.S. (2005) Host-specificity of Salmonella infections in animal species. In: Mastroeni, P., Maskell, D., editors. 'Salmonella' Infections: Clinical, Immunological and Molecular Aspects, Cambridge University Press. 57-80.

4. Uzzau, S., Leori, G.S., Petruzzi, V., Watson, P. R., Schianchi, G, Bacciu, D., Mazzarello, V., Wallis, T. S. and Rubino, S. (2001) Salmonella enterica Serovar-Host Specificity Does Not Correlate with the Magnitude of Intestinal Invasion in Sheep. Infect. Immun., 69(5):3092-3099.

5. Kingsley, R. and Baumler, A.J. (2000). Host adaptation and the emergence of infectious disease: the Salmonella paradigm. Mol. Microbiol. 36(5):1006-1014.
6. Bäumler, A.J., Tsolis, R.M., Ficht, T.A. and Adams, L.G. (1998) Evolution of host adaptation in Salmonella enterica. Infect. Immun. 66:4579-4587.

7. Retchless, A.C. and Lawrence, J. G. (2010) Phylogenetic incongruence arising from fragmented speciation in enteric bacteria. PNAS. 107 : 11453-11458.

8. Collazo, C.M. and Galan, J.E. (1997) The invasionassociated type-III protein secretion system in Salmonella-a review. Gene.192:51-59.

9. Hensel, M. (2000) Salmonella pathogenicity island 2. Mol. Microbiol.36:1015-1023.

10. Karasova, D., Sebkova, A., Havlickova, H., Sisak, F., Volf, J., Faldyna, M., Ondrackova, P., Kummer, V. and Rychlik, I. (2010) Influence of 5 major Salmonella pathogenicity islands on NK cell depletion in mice infected with Salmonella enterica serovar Enteritidis. BMC Microbiol. 10:75.

11. De Lappe, N. (2009) Salmonella Taxonomy. Version 1. Ref NSRLFM041, Dept of Medical Microbiology, Division of Clinical Microbiology, Galway University Hospitals. http:// www.nuigalway.ie/research/salmonella_lab/downloads/sal monella_taxonomy.pdf. Accessed on 5/06/2013.

12. Nnalue, N.A. (1991) Relevance of inoculation route to virulence of three Salmonella spp. strains in mice. Microb. Pathog. 11: 11-8.

13. Ellermeier, C. D. and Slauch, J. M. (2006) The Genus Salmonella In: Dworkin, M., Falkow, S., Rosenberg E., Schleifer, K.H, Stackebrandt, E., editors. The Prokaryotes A Handbook on the Biology of Bacteria, 3rd Edition, Springer Science and Business Media, LLC, 6: 123-158.

14. Clarke, R.C. and Gyles, C.L. (1993) Salmonella. In: Gyles, C.L., Thoen, C.O., editors. Pathogenesis of Bacterial Infections in Animals, 2nd edn. Iowa State University Press, Ames, Iowa. 133-153.

15. Uzzau, S., Brown D. J., Wallis, T., Rubino, S., Leori, G., Bernard S., Casadesus J., Platt, D. J. and Olsen, J. E. (2000) Host adapted serotypes of Salmonella enterica. Epidemiol. Infect. 125: 229-255.

16. Alpuche-Aranda, C. M., Berthiaume, E. P., Mock, B., Swanson, J. A. and Miller, S. I. (1995) Spacious phagosome formation within mouse macrophages correlates with Salmonella serotype pathogenicity and host susceptibility. Infect. Immun. 63: 4456-62.

17. Knodler, L. A. and Finlay, B. B. (2001) Salmonella and apoptosis; to live or let die? Microbes Infect. 3: 1321-1326.

18. Rubino, S., Leori, G., Rizzu, P., Erre, G., Colombo, M. M., Uzzau, S., Masala, G. and Cappuccinelli, P. (1993) TnphoA Salmonella abortusovis mutants unable to adhere to epithelial cells and with reduced virulence in mice. Infect. Immun. 61 (5): 1786-92.

19. Reynolds, J.D. and Morris, B. (1983) The evolution and involution of Peyer's patches in fetal and postnatal sheep. Eur. J. Immunol. 13: 627-35.

20. Watson, P. R., Galyov, E. E., Paulin, S. M., Jones, P. W. and Wallis, T. S. (1998) Mutation of invH, but not stn, reduces Salmonella-induced enteritis in cattle. Infect. Immun., 66:1432-8.

21. Pan, Z., Carter, B., Nunez-Garcia, J., Abuoun. M., Fookes, M., Ivens, A., Woodward, M.J. and Anjum, M.F. (2009) Identification of genetic and phenotypic differences associated with prevalent and non-prevalent Salmonella Enteritidis phage types: analysis of variation in amino acid transport. Microbiology. 155:3200-3213.

22. Gantois, I., Ducatelle, R., Pasmans, F., Haesebrouck, F. and Van Immerseel, F. (2008) Salmonella enterica serovar Enteritidis genes induced during oviduct colonization and egg contamination in laying hens. Appl Environ Microbiol. 74(21):6616-6622.

23. Guard, J., Shah, D.H., Morales, C.A. and Call, D.R. (2011) Evolutionary Trends Associated with Niche Specialization as Modelled by Whole Genome Analysis of Eggcontaminating Salmonella enterica serovar Enteritidis. In: Porwollik, S., editor. Salmonella: From Genome to Function. 
San Diego, CA: Caister Academic Press. 91-106.

24. Chiu, C.H., Tang, P., Chu, C., Hu, S., Bao, Q., Yu, J., Chou, Y.Y., Wang, H.S. and Lee, Y.S. (2005) The genome sequence of Salmonella enterica serovar Choleraesuis, a highly invasive and resistant zoonotic pathogen. Nucleic Acids Res. 33: 1690-1698.

25. McClelland, M., Sanderson, K.E., Clifton, S.W., Latreille, P., Porwollik, S., Sabo, A., Meyer, R., Bieri, T., Ozersky, P., McLellan, M., Harkins, C.R., Wang, C., Nguyen, C., Berghoff, A., Elliott, G., Kohlberg, S., Strong, C., Du, F., Carter, J., Kremizki, C., Layman, D., Leonard, S., Sun, H., Fulton, L., Nash, W., Miner, T., Minx, P., Delehaunty, K., Fronick, C., Magrini, V., Nhan, M., Warren, W., Florea, L., Spieth, J. and Wilson, R.K. (2004) Comparison of genome degradation in Paratyphi A and Typhi, humanrestricted serovars of Salmonella enterica that cause typhoid. Nat. Genet. 36: 1268-1274.

26. Holt, K.E., Thomson, N.R., Wain, J., Langridge, G.C., Hasan, R., Bhutta, Z.A., Quail, M.A., Norbertczak, H., Walker, D., Simmonds, M.,White, B., Bason, N., Mungall, K., Dougan, G. and Parkhill, J. (2009) Pseudogene accumulation in the evolutionary histories of Salmonella enterica serovars Paratyphi A and Typhi. BMC Genomics. 10:36.

27. Thomson, N.R., Clayton, D.J., Windhorst, D., Vernikos, G., Davidson, S., Churcher, C., Quail, M.A., Stevens, M., Jones, M.A., Watson, M., Barron, A., Layton, A., Pickard, D., Kingsley, R.A., Bignell, A., Clark, L., Harris, B., Ormond, D., Abdellah, Z., Brooks, K., Cherevach, I., Chillingworth, T., Woodward, J., Norberczak, H., Lord, A., Arrowsmith, C., Jagels, K., Moule, S., Mungall, K., Sanders, M., Whitehead, Chabalgoity, J.A., Maskell, D., Humphrey, T., Roberts, M., Barrow, P.A., Dougan, G., and Parkhill, J. (2008) Comparative genome analysis of Salmonella Enteritidis PT4 and Salmonella Gallinarum 287/91 provides insights into evolutionary and host adaptation pathways. Genome Res. 18: 1624-1637.

28. Kingsley, R.A., Msefula, C.L., Thomson, N.R., Kariuki, S., Holt, K.E., Gordon, M.A., Harris, D., Clarke, L., Whitehead, S., Sangal ,V., Marsh, K., Achtman, M., Molyneux, M.E., Cormican, M., Parkhill, J., MacLennan, C.A., Heyderman, R.S. and Dougan, G. (2009) Epidemic multiple drug resistant Salmonella Typhimurium causing invasive disease in subSaharan Africa have a distinct genotype. Genome Res. 19: 2279-2287.

29. Kisiela, D.I., Chattopadhyay, S., Libby, S.J., Karlinsey, J.E., Fang, F.C., Tchesnokova, V., Kramer, J.J, Beskhlebnaya, V., Samadpour, M., Grzymajlo, K., Ugorski, M., Lankau, E.W., Mackie, R.I., Clegg, S. and Sokurenko, E.V. (2012) Evolution of Salmonella enterica Virulence via Point Mutations in the Fimbrial Adhesin. PLoS Pathog. 8(6): e1002733.

30. Olsen, J.E., Hoegh-Andersen, K.H., Casadesús, J., Rosenkranzt, J., Chadfield, M.S. and Thomsen, L.E. (2013)
The role of flagella and chemotaxis genes in host pathogen interaction of the host adapted Salmonella enterica serovar Dublin compared to the broad host range serovar $S$. Typhimurium. BMC Microbiology. 13:67.

31. Rabsch, W., Andrews, H. L., Kingsley, R. A., Prager, R., Tschäpe, H., Adams, L. G., and Baumler, A. J. (2002) Salmonella enterica serotype Typhimurium and its hostadapted variants. Infect. Immun. 70: 2249-2255.

32. Heithoff, D.M, Shimp, W.R., Lau, P.W., Badie. G., Enioutina, E.Y., Daynes, R. A., Byrne, B.A., House, J.K. and Mahan, M.J. (2008) Human Salmonella Clinical Isolates Distinct from Those of Animal Origin. Appl. Environ Microbiol. 74(6): 1757-1766.

33. Guo, A., Cao, S., Tu, L., Chen, P., Zhang, C., Jia, A., Yang, W., Liu, Z., Chen, H. and Schifferli, D.M. (2009) FimH alleles direct preferential binding of Salmonella to distinct mammalian cells or to avian cells. Microbiology. 155: 1623-33.

34. De Jong, H.K., Parry, C.M, van der Poll, T. and Wiersinga, W.J. (2012). Host-Pathogen Interaction in Invasive Salmonellosis. PLOs Pathogens. 8(10): e1002933.

35. Eswarappa, S.M., Janice, J., Nagarajan, A.G., Balasundaram, S.V., Karnam G, Dixit, N.M. and Chakravortty, D. (2008) Differentially Evolved Genes of Salmonella Pathogenicity Islands: Insights into the Mechanism of Host Specificity in Salmonella. PLoS ONE 3(12): e3829.

36. Eswarappa, S.M., Janice, J., Balasundaram, S.V., Dixit, N.M. and Chakravortty, D. (2009) Host-specificity of Salmonella enterica serovar Gallinarum: insights from comparative genomics. Infect. Genet. Evol. 9: 468-473.

37. Liu, W.Q., Feng, Y., Wang, Y., Zou, Q.H., Chen, F., Guo, J.T., Peng, Y.H., Jin, Y., Li, Y.G., Hu, S.N., Johnston, R.N., Liu, G.R. and Liu, S.L. (2009) Salmonella Paratyphi C: Genetic Divergence from Salmonella Choleraesuis and Pathogenic Convergence with Salmonella Typhi. PLoS ONE 4(2):e4510.

38. Chen, H.M., Wang, Y., Su, L.H. and Chiu, C.H. (2013) Nontyphoid Salmonella Infection: Microbiology, Clinical Features, and Antimicrobial Therapy. Pediatrics and Neonatology. 54(3): 147-152.

39. Scheelings, T.F., Lightfoot, D. and Holz, P. (2011) Prevalence of Salmonella in Australian reptiles. J Wildl. Dis. 47: $1-11$.

40. Richardson, E.J., Limaye, B., Inamdar, H., Datta, A., Manjari, K.S., Pullinger, G.D., Thomson, N.R., Joshi, R.R., Watson, M. and Stevens, M.P. (2011) Genome Sequences of Salmonella enterica Serovar Typhimurium, Choleraesuis, Dublin, and Gallinarum Strains of Well-Defined Virulence in Food-Producing Animals. J. Bacteriol. 193(12):31623163.

41. Stevens, M.P., Humphrey, T.J. and Maskell, D.J. (2009) Molecular insights into farm animal and zoonotic Salmonella infections. Phil. Trans. R. Soc. B. 364: 27092723. 